nướu của phục hồi xoang II sử dụng composite Clearfil - APX có liên quan đến phương pháp đặt chất dán. Sự khác biệt về vi kẽ của hai kỹ thuật còn phụ thuộc vào loại Composite sử dụng để phục hồi.

\section{TÀI LIỆU THAM KHẢO}

1. Davidson CL, De Gee AJ, "Relaxation of polymerization contraction stresses by flow in dental composites",J Dent Res 1984;63:146-148.

2. Platt JA, Almeida J, Gonzales-Cabezas $C$, Rhodes B, Moore BK, "The effect of double adhesive application on the shear bond strength to dentin of compomers using three one bottle adhesive systems",Oper Dent 2001;26: 313-317.
3. Rajni N, Pallavi $S$, Naveen M (2015), "Influence of double application technique on the bonding effectiveness of self-etch adhesive systems", Microscopy research and technique 78:489-494 (2015)

4. Rueggeberg FA, Margeson DH, "The effect of oxygen inhibition on an unfilled/filled composite

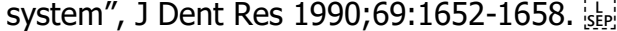

5. Simone deliperi, David N. Bardwell, "Microleakage of a microhybrid composite resin using three different adhesive placement techniques",] adhes Dent

6. Van Meerbeek B, Vargas M, Inoue S, Yoshida Y, Peumans M, Lambrechts P, Vanherle G, "Ádhesives and cements to promote preservation dentistry",Oper Dent 2001;(suppl 6):119-144.

\title{
NGHIÊN CỨU MộT Số KÍCH THƯớC ổ CốI, GÓC NGHIÊNG TRƯớC CỦA Cổ XƯƠ'NG ĐÙI VÀ GÓC NGHIÊNG KẾT HỢP TRÊN PHIM CHỤP CẮT LỚP VI TÍNH
}

\section{TÓM TẮT}

Qua nghiên cứu trên 54 phim chụp cắt lớp vi tính của 54 bệnh nhân không có bệnh lý khớp háng ở ít nhất 1 bên tại Bệnh viện Đại học Y Hà Nội, chúng tôi thu được kết quả như sau: Giá trị góc nghiêng ngoài ổ cối là: $37.48 \pm 4.95^{\circ}$; Giá trị góc nghiêng trước ổ cối là: $17.2 \pm 5.81^{\circ}$; Giá trị góc nghiêng trước của cổ xương đùi là: $12.03 \pm 7.32^{\circ}$; Giá trị góc nghiêng kết hợp là: $29.23 \pm 9.07^{\circ}$

Từ khóa: Ổ cối, góc nghiêng trước cổ xương đùi, góc nghiêng kết hợp.

\section{SUMMARY}

\section{RESEARCH ON ACETABULLAR PARAMETERS, FEMORAL AND COMBINED ANTEVERSION ANGLES ON COMPUTED- TOMOGRAPHY SCAN}

54 computed-tomography scans of 54 Vietnamese adults with at least 1 non-pathological hip at Hanoi Medical University Hospital are used on our research. The results are: Acetabullar inclination angle: $37.48 \pm$ 4.95; Acetabullar anteversion angle: $17.2 \pm 5.81^{\circ}$; Femoral anteversion angle: $12.03 \pm 7.32^{\circ}$; Combined anteversion: $29.23 \pm 9.07^{\circ}$.

Keyword: Acetabulum, femoral anteversion, combined anteversion.

\footnotetext{
${ }^{1}$ Trường Đại học Y Hà Nội

²Bênh viện Đại học Y Hà Nội

Chịu trách nhiệm chính: Trần Lê Đình Duy

Email: tranledinhduy.hmu@gmail.com

Ngày nhận bài: 7/3/2021

Ngày phản biện khoa học: 9/4/2021

Ngày duyệt bài: 5/5/2021
}

Trần Lê Đình Duy ${ }^{1}$, Trần Sinh Vương ${ }^{1}$, Nguyễn Văn Hoạt ${ }^{2}$, Ngô Xuân Khoa ${ }^{1}$.

\section{I. ĐĂT VẤN ĐỀ}

Những thông tin về các kích thước bình thường của ổ cối, góc nghiêng trước của cổ xương đùi nói riêng và khớp háng nói chung có vai trò rất quan trọng trong việc lựa chọn khớp háng nhân tạo phù hợp với bệnh nhân cũng như giúp phẫu thuật viên dễ dàng đánh giá kết quả hậu phẫuhay khớp háng [1]. Với mục đích cung cấp thêm số liệu bình thường của các kích thước ổ cối và các góc nghiêng trên phim chụp cắt lớp vi tính, đồng thời góp phần giúp các nhà ngoại khoa và các bác sĩ có thêm số liệu tham khảo trong thực hành lâm sàng, chúng tôi tiến hành nghiên cứu với mục tiêu: Khảo sát các kích thước góc nghiêng ngoài ổ cối, góc nghiêng trước ố cối, góc nghiêng trước của cổ xương đưii, góc nghiêng kêt hợp trên phim chụp cắt lớp vi tính ở người Việt Nam trưởng thành.

\section{II. ĐỐI TƯỢNG VÀ PHƯƠNG PHÁP NGHIÊN CỨU}

2.1. Đối tượng nghiên cứu. Phim chụp cắt lớp vi tính qua khung chậu và xương đùi được lưu trữ trong hệ thống cơ sở dữ liệu hình ảnh (Picture Archiving and Communication System PACS) của 54 bệnh nhân độ tuổi từ 25 đến 96 (tuổi trung bình: 61士16.62) không mắc bệnh lý cơ - xương - khớp (viêm khớp, hoại tử chỏm xương đùi, biến dạng khớp háng ...) ở một hoặc cả hai bên khớp tại Khoa Chẩn đoán hình ảnh, Bệnh viện Đại học Y Hà Nội, trong thời gian từ tháng 08/2018 đến tháng 04/2020. 


\subsection{Phương pháp nghiên cứu}

- Góc nghiêng ngoài ổ cối được xác định trên lát cắt vành (coronal) đi qua tâm chỏm xương đùi, góc nghiêng trước ổ cối được xác định trên lát cắt trục (axial) mà tai đó, kích thước từ rìa trước đến rìa sau ổ cối là lớn nhất, góc nghiêng trước của cổ xương đùi được đo bằng phương pháp lát cắt axial đơn (single-sliced axial), góc nghiêng kết hợpđược tính bằng bằng tổng góc nghiêng trước ổ cối và góc nghiêng trước của cổ xương đùi.

- Số liệu về các kích thước đầu trên xương đùi được đo trên cơ sở dữ liệu PACS, ghi lại bằng bệnh án nghiên cứu và xử lý bằng phần mềm SPSS 20.0 và Microsoft Excel Office 2016. Tính chuẩn của các biến được kiểm tra bằng kiểm định Komogrov-Smirnoff. Để so sánh trung bình giữa các biến với nhau, kiểm định t-test và t-test ghép cặp được sử dụng với các biến chuẩn, test kiểm định Mann-Whitney-U được sử dụng với các biến không chuẩn. Hệ số tương quan Spearman (đối với biến chuẩn) và Pearson (đối với biến không chuẩn) được sử dụng để khảo sát mối tương quan giữa các biến với nhau.

\section{KẾT QUẢ NGHIÊN CỨU VÀ BÀN LUÂN}

\subsection{So sánh các kích thước giữa hai giới}

Bảng 1. Các kích thước ổ côi và các góc nghiêng theo giới

\begin{tabular}{|c|c|c|c|c|c|}
\hline & & $\begin{array}{l}\text { Góc nghiêng } \\
\left.\text { ngoài ổ cối ( }{ }^{\circ}\right)\end{array}$ & $\begin{array}{l}\text { Góc nghiêng } \\
\left.\text { trước ố cối ( }{ }^{\circ}\right)\end{array}$ & $\begin{array}{l}\text { Góc nghiêng } \\
\text { trước cổ } \\
\left.\text { xương đù̀i( }{ }^{\circ}\right)\end{array}$ & $\begin{array}{l}\text { Góc nghiêng } \\
\text { kết hợp ( }\left(^{\circ}\right)\end{array}$ \\
\hline & Chung & $37.48 \pm 4.95$ & $17.2 \pm 5.81$ & $12.03 \pm 7.32$ & $29.23 \pm 9.07$ \\
\hline Nghien cưu & Nam & $36.89 \pm 5.17^{*}$ & $16.08 \pm 5.65^{*}$ & $10.47 \pm 6.11^{*}$ & $26.54 \pm 8.03^{*}$ \\
\hline & Nữ & $39.25 \pm 3.8^{*}$ & $20.51 \pm 5.04 *$ & $16.66 \pm 8.69 *$ & $37.17 \pm 7.22^{*}$ \\
\hline Trân Trung & Chung & $38.5 \pm 3.9$ & $17.2 \pm 7.0$ & $13.7 \pm 10.4$ & $30.8 \pm 13.2$ \\
\hline Dũng và cs. & Nam & $38.6 \pm 3.8$ & $16.3 \pm 7.1$ & $13.3 \pm 11.7$ & $29.6 \pm 14.8$ \\
\hline [2] & Nũ̃ & $38.3 \pm 4.3$ & $18.7 \pm 6.8$ & $14.3 \pm 7.8$ & $33.0 \pm 9.8$ \\
\hline Aditya V. & Chung & - & $19.1 \pm 5.0$ & $8.0 \pm 4.7$ & $27.1 \pm 6.3$ \\
\hline Maheshwari & Nam & - & $17.3^{*}$ & 7.3 & $24.6 *$ \\
\hline và cs. [3] & Nũ̃ & - & $20.8^{*}$ & 8.7 & $29.5^{*}$ \\
\hline & Chung & - & $18.79 \pm 5.30$ & $10.62 \pm 9.02$ & - \\
\hline Na & Nam & - & $18.27 \pm 5.22 *$ & $9.31 \pm 8.58^{*}$ & \\
\hline & Nữ & - & $20.44 \pm 5.26^{*}$ & $14.76 \pm 9.13^{*}$ & \\
\hline
\end{tabular}

*Khác biệt ở hai giới có ý nghĩa thống kê với $\mathrm{p}<0.05$

Từ kết quả bảng 1,góc nghiêng ngoài ổ cối ở nam giới $\left(36.89 \pm 5.17^{\circ}\right)$ nhỏ hơn nữ giới $(39.25$ $\left.\pm 3.8^{\circ}\right)$, góc nghiêng trước ổ cối ở nam giới $\left(16.08 \pm 5.65^{\circ}\right)$ nhỏ hơn nữ giới $\left(20.51 \pm 5.04^{\circ}\right)$, góc nghiêng trước của cổ xương đùi ở nam giới $\left(10.47 \pm 6.11^{\circ}\right)$ nhỏ hơn nữ giới $\left(16.66 \pm 8.69^{\circ}\right)$, góc nghiêng kết hợp ở nam giới $\left(26.54 \pm 8.03^{\circ}\right)$ nhỏ hơn nữ giới $\left(37.17 \pm 7.22^{\circ}\right)$. Những sự khác biệt này có ý nghĩa thống kê với $p<0.05$.

Kết quả của nghiên cứu này này khác với kết quả nghiên cứu của Trần Trung Dũng và cộng sự, trong đó không có sự khác biệt các kích

thước khớp háng giữa hai giới. Trong nghiên cứu của Aditya V. Maheshwari và cộng sự, không có sự khác biệt về giá trị của góc nghiêng trước của cổ xương đùi ở hai giới $\left(7.3^{\circ}\right.$ ở nam giới và $8.7^{\circ}$ ở nữ giới), có sự khác biệt về góc nghiêng trước ổ cối (17.3 ở nam giới và $20.8^{\circ}$ ở nữ giới) và góc nghiêng kết hợp (24.6 ở nam và $29.5^{\circ}$ ở nữ). Kết quả nghiên cứu của Nanjiang và cộng sự cho thây có sự khác biệt giữa hai giới về giá trị góc nghiêng trước ổ cối $\left(18.27 \pm 5.22^{\circ}\right.$ ở nam giới và $20.44 \pm 5.26^{\circ}$ ở nữ giới) và góc nghiêng trước của cổ xương đùi $\left(9.31 \pm 8.58^{\circ}\right.$ ở nam giới và $14.76 \pm 9.13^{\circ}$ ở nữ giới).

\subsection{So sánh cáckích thước khớp háng giữa bên khớp khảo sát}

Bảng 2. Các kích thước ổ cối và các góc nghiêng so với nghiên cứu khác.

\begin{tabular}{|c|c|c|c|c|c|}
\hline & $\begin{array}{c}\text { Góc nghiêng } \\
\text { ngoài ổ cối } \\
\left({ }^{\circ}\right)\end{array}$ & $\begin{array}{l}\text { Góc nghiêng } \\
\left.\text { trước ổ cối ( }{ }^{\circ}\right)\end{array}$ & $\begin{array}{l}\text { Góc nghiêng } \\
\text { trước cổ } \\
\text { xương đùi }\left({ }^{\circ}\right)\end{array}$ & $\begin{array}{l}\text { Góc nghiêng } \\
\text { kết hợp ( }\left(^{\circ}\right)\end{array}$ \\
\hline \multirow{3}{*}{$\begin{array}{l}\text { Nghiên cứu } \\
\text { này }\end{array}$} & Chung & $37.48 \pm 4.95$ & $17.2 \pm 5.81$ & $12.03 \pm 7.32$ & $29.23 \pm 9.07$ \\
\hline & Trái & $36.95 \pm 4.87$ & $16.79 \pm 5.87$ & $12.15 \pm 7.38$ & $28.94 \pm 8.61$ \\
\hline & Phải & $38.01 \pm 5.03$ & $17.59 \pm 5.79$ & $11.91 \pm 7.34$ & $29.5 \pm 9.58$ \\
\hline
\end{tabular}




\begin{tabular}{|c|c|c|c|c|c|}
\hline \multirow{2}{*}{$\begin{array}{c}\text { Aditya V. } \\
\text { Maheshwari } \\
\text { và cs. }\end{array}$} & Chung & - & $19.1 \pm 5.0$ & $8.0 \pm 4.7$ & $27.1 \pm 6.3$ \\
\cline { 2 - 6 } & Trái & - & 18.9 & $7.4^{*}$ & 27.6 \\
\cline { 2 - 6 } Nan Jiang & Phải & - & 19.2 & $8.7^{*}$ & 26.6 \\
\cline { 2 - 6 } và cs. & Chung & - & $18.79 \pm 5.30$ & $10.62 \pm 9.02$ & - \\
\cline { 2 - 6 } & Phái & - & $18.43 \pm 5.21^{*}$ & $10.93 \pm 9.13$ & - \\
\hline
\end{tabular}

*Khác biệt hai bên có ý nghĩa thống kê với $p<0.05$

Từ kết quả bảng 2, không có sự khác biệt giữa góc nghiêng ngoài ổ cối, góc nghiểng trước ổ cối, góc nghiêng trước của cổ xương đùi, góc nghiêng kết hợp giữa hai bên khớp được khảo sát.

Kểt quả nghiên cứu của Aditya V. Maheshwari khi so sánh các kích thước khớp háng hai bên cho thấy: Có sự khác biệt giữa góc nghiêng trước của cổ xương đùi $\left(7.4^{\circ}\right.$ đối với bên phải và $8.7^{\circ}$ đối với bên trái), không có sự khác biệt góc nghiêng trước ổ cối (19.20 đối với bên phải và $18.9^{\circ}$ với bên trái) và góc nghiêng kết hợp (26.6 ở bên phải và $27.6^{\circ}$ ở bên trái). Trong nghiên cứu của Nanjiang và cộng sự, có sự khác biệt giá

trị góc nghiêng trước ổ cối ở hai bên khớp $\left(18.43 \pm 5.21^{\circ}\right.$ ở bên trái so với $19.10 \pm 5.38^{\circ}$ ở bên phải), không có sự khác biệt ở hai bên khớp với góc nghiêng trước của cổ xương đùi (10.93 $\pm 9.13^{\circ}$ ở bên trái và $10.41 \pm 8.85^{\circ}$ ở bên phải). Sự khác biệt giữa các kích thước khớp háng hai bền có thể do ưu thế bên thuận (leg dominance). Chân thuận, thường là bên phải, có cường độ, biên độ vận động lớn hơn nên các kích thước và góc của cổ, chỏm xương đùi và ổ cối bên thuận có xu hướng nhỏ hơn do trong quá trình phát triển, chân bên thuận chịu lực lớn hơn. [5]

\subsection{Tương quan giữa các kích thước đâu trên xương đùi}

Bảng 4. Tương quan giữa kích thước ổ côi và các góc nghiêng

\begin{tabular}{|c|c|c|c|c|}
\hline Kích thước & $\begin{array}{c}\text { Góc } \\
\text { nghiêng } \\
\text { trước ổ cối }\end{array}$ & $\begin{array}{c}\text { Góc } \\
\text { nghiêng } \\
\text { ngoài ổ cối }\end{array}$ & $\begin{array}{c}\text { Góc nghiêng } \\
\text { trước của cổ } \\
\text { xương đùi }\end{array}$ & $\begin{array}{c}\text { Góc } \\
\text { nghiêng } \\
\text { kết hợp }\end{array}$ \\
\hline Góc nghiêng trước ổ cối & 1 & & & \\
\hline Góc nghiêng ngoài ổ cối & 0.01 & 1 & & \\
\hline Góc nghiêng trước của cổ xương đùi & -0.66 & -0.59 & 1 & 1 \\
\hline Góc nghiêng kết hợp & $0.592^{*}$ & -0.047 & $0.769^{*}$ & 1 \\
\hline
\end{tabular}

* Sự tương quan có ý nghĩa thống kê với $p<0.01$

Từ kết quả bảng 4, Góc nghiêng kết hợp có tương quan đồng biến với góc nghiêng trước của CXĐ và góc nghiêng trước ổ cối với hệ số tương quan lân lượt là 0.592 và 0.769 .Kết quả Leonard T. Buller cho thấy có sự tương quan giữa góc nghiêng trước của $C X \bigoplus$ và góc nghiêng ngoài ổ cối, giữa góc nghiêng trước của $C X \bigoplus$ và góc nghiêng trước ổ cối. [6] Kết quả nghiên cưu trên 47 người có khớp háng không tổn thương và 39 người viêm khớp háng của và cộng sự cho thấy không có sự tương quan giữa góc nghiêng trước của CXĐ và góc nghiêng trước ổ cối, và nếu tổn tại sự tương quan giữa hai góc này sẽ có thể dẫn đến viêm khớp háng. [7]

\section{KẾT LUẬN}

- Giá trị góc nghiêng trước của cổ xương đùi là $12.03 \pm 7.32^{\circ}$, trong đó có sự khác biệt giá trị kích thước này giữa nhóm tuổi dưới 60 và từ 60 trở lên.

- Giá trị góc nghiêng trước ảa ổ cối là 17.2 土 5.81․

- Giá trị góc nghiêng ngoài của ổ cối là 37.48 $\pm 4.95^{\circ}$.
- Giá trị của góc nghiêng kết hợp là $29.23 \pm 9.07^{\circ}$.

\section{TÀI LIÊU THAM KHẢO}

1. Dimitriou.D., Tsai.T.-Y., Yue.B., và cộng sự. (2016). Side-to-side variation in normal femoral morphology: 3D CT analysis of 122 femurs. Orthopaedics \& Traumatology: Surgery \& Research, 102(1), p. 91-97.

2. Tran Trung.D., Pham Trung.H., Truong Cong.M., và cộng sự. (2019). The anatomical characteristics of Vietnamese adult hip joint: a multiplanar reconstruction computer tomographic study. Muscle Ligaments and Tendons Journal, 09(01), p. 165.

3. Maheshwari.A. V., Zlowodzki.M.P., Siram.G., và cộng sự. (2010). Femoral neck anteversion, acetabular anteversion and combined anteversion in the normal Indian adult population: A computed tomographic study. Indian Journal of Orthopaedics, 44(3), p. 277-282.

4. Jiang.N., Peng.L., Al-Qwbani.M., và cộng sứ, (2015). Femoral version, neck-shaft angle, and acetabular anteversion in Chinese Han population: a retrospective analysis of 466 healthy adults. Medicine, 94(21), p. e891.

5. Gilligan.I., Chandraphak.S., và Mahakkanukrauh.P. (2013). Femoral neckshaft angle in humans: Variation relating to 
climate, clothing, lifestyle, sex, age and side. Journal of Anatomy, 223(2), p. 133-151.

6. Buller.L.T., Rosneck.J., Monaco.F.M., và cộng sự. (2012). Relationship between proximal femoral and acetabular alignment in normal hip joints using 3-dimensional computed tomography. American Journal of Sports Medicine, 40(2), p.
367-375.

7. Reikeråls.0., Bjerkreim.I., và Kolbenstvedt.A. (1983). Anteversion of the Acetabulum and Femoral Neck in Normals and in Patients with Osteoarthritis of the Hip. Acta Orthopaedica Scandinavica, 54(1), p. 18-23.

\title{
KIẾN THỨC CỦA SINH VIÊN ĐIỀU DƯỡNG NĂM CUỐI TRƯỜNG ĐẠI HỌC ĐIỀU DƯỠNG NAM ĐINH VỀ DƯ PHÒNG PHƠI NHIỄM VỚI MÁU, DICH CO' THỂ
}

\author{
Vũ Thị Là ${ }^{1}$, Hoàng Thị Minh Thái ${ }^{1}$, \\ Nguyễn Mạnh Dũng1, Phạm Văn Tùng ${ }^{1}$, Nguyễn Thị Lĩnh ${ }^{1}$
}

TÓM TẮT

Mục tiêu: mô tả kiến thức của sinh viên điều dưỡng năm cuối Trường Đại học Điều dưỡng Nam Định về phòng ngừa phơi nhiễm với máu, dịch cơ thể. Đối tượng và phương pháp: Sinh viên đại hợc điều dưỡng chính quy năm cuối được lựa chon ngẫu nhiên và trả lời câu hỏi bằng phiếu khảo sát. Kết quả: Điểm trung bình khái niệm và phương thức; chăm sóc sức khỏe cơ bản; biện pháp phòng ngừa; sơ cứu tại chỗ; báo cáo và lập biên bản; đánh giá nguy cơ và nguồn lây; điều trị dự phòng sau phơi nhiễm lần lượt là 8,38; 8,$36 ; 9,38 ; 7,84 ; 8,13 ; 7,07$ và 5,99điểm. Kết luận: Kiến thức phòng ngừa phơi nhiếm với máu, dịch tiết của sinh viên điều dưỡng Nam Đinh đang ở mức độ trung bình với điểm trung bình chung là 7,71 \pm 0,71điểm. Các lĩnh vực sinh viên đang có kiến thức ở mức trung bình là sơ cứu tại chỗ và đánh giá nguồn lây nhiếm

Tư khóa: Kiến thức, sinh viên điều dưỡng, phơi

\section{SUMMARY}

FINAL-YEAR NURSING STUDENTS' KNOWLEDGE OF PREVENTING EXPOSURE

TO BLOOD AND BODY FLUIDS IN NAM DINH UNIVERSITY OF NURSING

Objective: Describe final-year nursing students' knowledge of preventing exposures to blood and body fluidsin Nam Dinh University of Nursing. Subjects and method: Finalyear full-time nursing students were randomly selected to answerquestionnaires. Results: mean scoresof concept and mode; fundamental health care; prevention measures; emergency first-aid; reporting and making a report; assessment of risk and the source of infection are in turn 8.38; 8.36; 9.38; 7.84; 8.13; 7.07 and 5.99points. Conclusion: Final-year nursing students'

${ }^{1}$ Trường ĐH Điều dưỡng Nam Định Chịu trách nhiệm chính: Vũ Thị Là Email: vula_ynd@yahoo.com.vn Ngày nhận bài: 21/3/2021

Ngày phản biên khoa học: 25/4/2021 Ngày duyệt bài: 6/5/2021 knowledge of preventing exposure to blood and body fluidin Nam Dinh University of Nursing is moderate with the mean score is $7.71 \pm 0.71$ points $/ 10$ points. The domainsin which students' knowledge is at an average level are emergency first-aid and assessmentof infection source.

Keyword: Knowledge, nursing student, Occupational exposure.

\section{I. ĐĂT VẤN ĐỀ}

Thực hành lâm sàng là một khía cạnh chuyên sâu của chương trình đào tạo và là một thành phân cốt Iõi trong giáo dục và đào tạo điêu dưỡng hiện nay. Đào tạo thực hành lâm sàng diễn ra trong các môi trường lâm sàng khác nhau, với mục đích rèn luyện kỹ năng tư duy và kỹ năng ra quyết định lâm sàng cũng như phát triển năng lực thực hành nghề nghiêp điều dưỡng. Tuy nhiên, môi trường lâm sàng tại bệnh viện luôn tiềm ẩn các yếu tố rủi ro nghề nghiệp như môi trường độc hại, các loại thuốc hóa trị, bạo lực, tổn thương do kim tiêm/vật sắc nhọn, các vấn đề về da, dị ứng latex, các bênh truyên nhiễm lây lan qua đường máu/dịch tiết, các vấn đề về sức khỏe tâm thần. Đặc biệt, phơi nhiễm với các bệnh truyền nhiễm qua đường máu hoặc dịch cơ thể đang là một vấn đề cân được quan tấm ở sinh viên điều dưỡng.

Phơi nhiễm với máu, dich cơ thể ở sinh viên điêu dưỡng là tai nạn rủi ro trong quá trình thực hành nghề[8]. Các phơi nhiễm có thể xảy ra đối với sinh viên điều dưỡng chủ yếu là tổn thương qua da từ kim tiêm hoăc vât sắc nhọn có dính máu, dịch tiết của người nhiễm hoặc máu, dịch cơ thể của người bị nhiễm tiếp xúc với da tổn thương hoặc bắn vào niêm mạc[3],[5]. Hậu quả của việc bị phơi nhiễm với máu, dịch cơ thể làm tăng nguy cơ lo âu, căng thẳng của sinh viên, nghiêm trọng hơn là nguy cơ nhiếm các tác nhân gây bệnh qua đường máu như: HBV, HCV, 